\title{
Self Assembled Nanostructure Networks on Surfaces of Layered Crystals
}

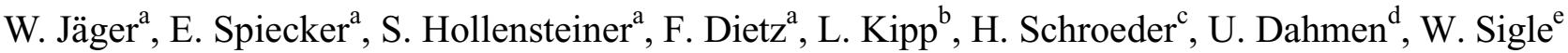 \\ ${ }^{a}$ Faculty of Engineering, Christian-Albrechts-University, Kaiserstrasse 2, 24143 Kiel, Germany \\ ${ }^{\mathrm{b}}$ IEAP, Christian-Albrechts-University Kiel, Ohlshausenstrasse 40, 24098 Kiel, Germany \\ ${ }^{\mathrm{c}}$ Institute of Solid State Research, Research Centre Jülich, 52425 Jülich Germany \\ d NCEM, Lawrence Berkeley National Laboratory, Berkeley, CA94720, USA \\ ${ }^{\mathrm{e}}$ Max-Planck-Institut für Metallforschung, Heisenbergstr.3, 70569 Stuttgart, Germany
}

The self assembly of planar network nanostructures, which develop during the early stages of metal deposition on initially smooth surfaces of layered crystal compounds, may serve the development of new nanoscale materials. Using deposition of copper onto layered $\mathrm{VSe}_{2}$ crystals as model system, the metal-induced surface phenomena have been investigated combining techniques of high-resolution and analytical TEM (including spatially-resolved ELNES, STEM and EDXS measurements), SEM, and atomic force microscopy. Large nanostructures of roof-like morphology (lateral dimensions $>$ $100 \mathrm{~nm}$ ), which consist of two strands of crystalline material, and interconnected hexagonal networks of nanostructures with smaller lateral dimensions ( several $10 \mathrm{~nm})$, predominantly aligned along low-index substrate directions, are observed to form on the substrate surfaces [1-4]. The nanostructures form as result of compressive strain built up during metal intercalation into the surface layer upon vacuum deposition [3]. Observations of nearly contiguous thin surface layers of a copper-rich crystalline intercalation phase, present in three orientation domains, confirm this conclusion [4]. The self assembled nanostructures and the surface phase layers depict different $\mathrm{Cu}$ $\mathrm{L}_{2,3}$ electron loss near-edge structures indicating that copper is incorporated with different bonding states [5]. In order to explore the use for potential nanoscale engineering of interconnected nanowires on surfaces, first investigations of the effects of deposition of (non-intercalating) gold on surface nanostructures have been performed, demonstrating a template effect by the formation of aligned metal aggregates.

References

[1] S. Hollensteiner et al., Mater. Sci. Eng. C 23 (2003) 171.

[2] E. Spiecker, Microsc. Microanal. 9(3) (2003) 300.

[3] E. Spiecker et al., Microsc. Microanal. (2004), accepted.

[4] S. Hollensteiner et al., Applied Surface Science 241 (2005) 49.

[5] S. Hollensteiner et al., Z. Metallkunde (2005), submitted

[6] This work was supported by the German Research Council (DFG contract FOR 353/2-1). 

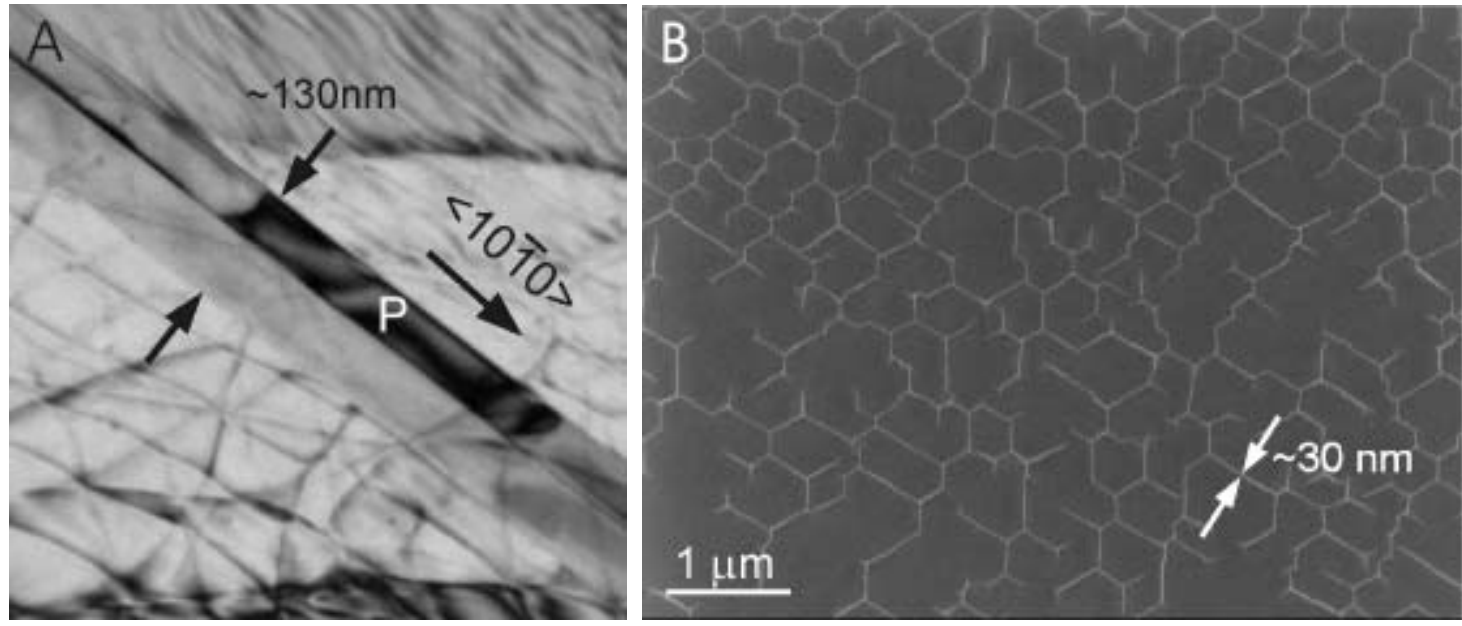

Fig.1 A: TEM bright-field micrograph of a larger nanostructure (P crystalline platelet) B: SEM image of hexagonal networks of small nanostructures formed by $\mathrm{Cu}$ deposition on a $\mathrm{VSe}_{2}$ surface.
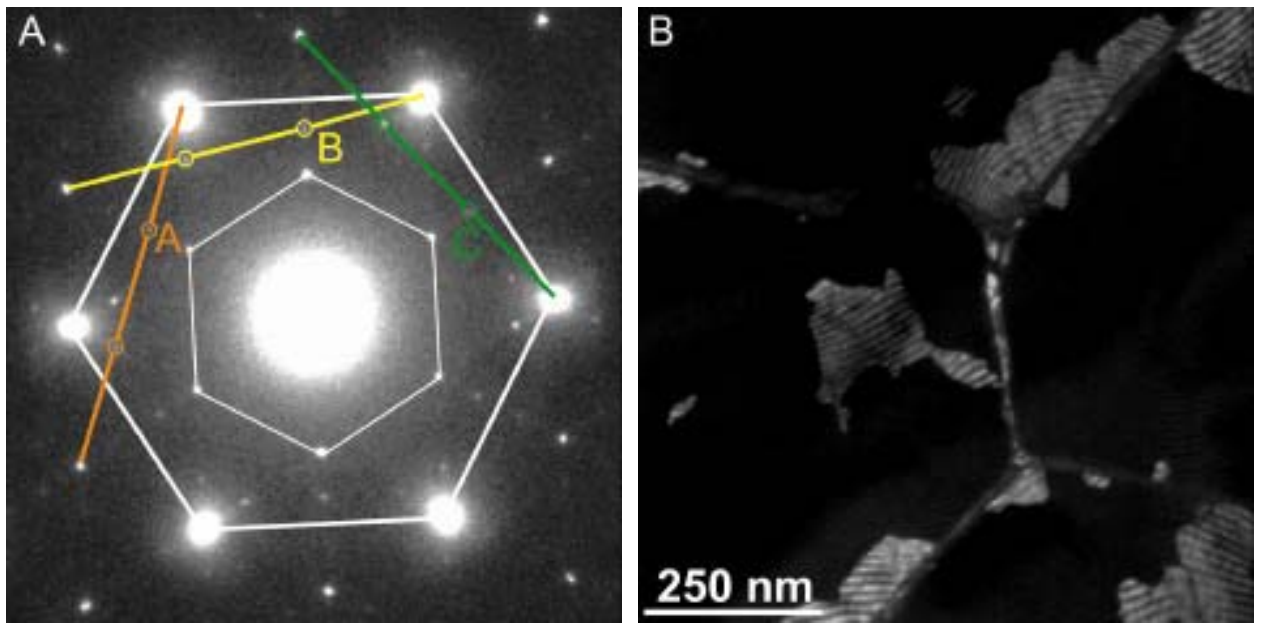

Fig.2 Cu-rich crystalline intercalation phase. A: [0001] zone-axis SAED pattern with layer reflections due to 3 orientation domains (A, B, C). B: TEM DF micrograph of individual orientation domain.
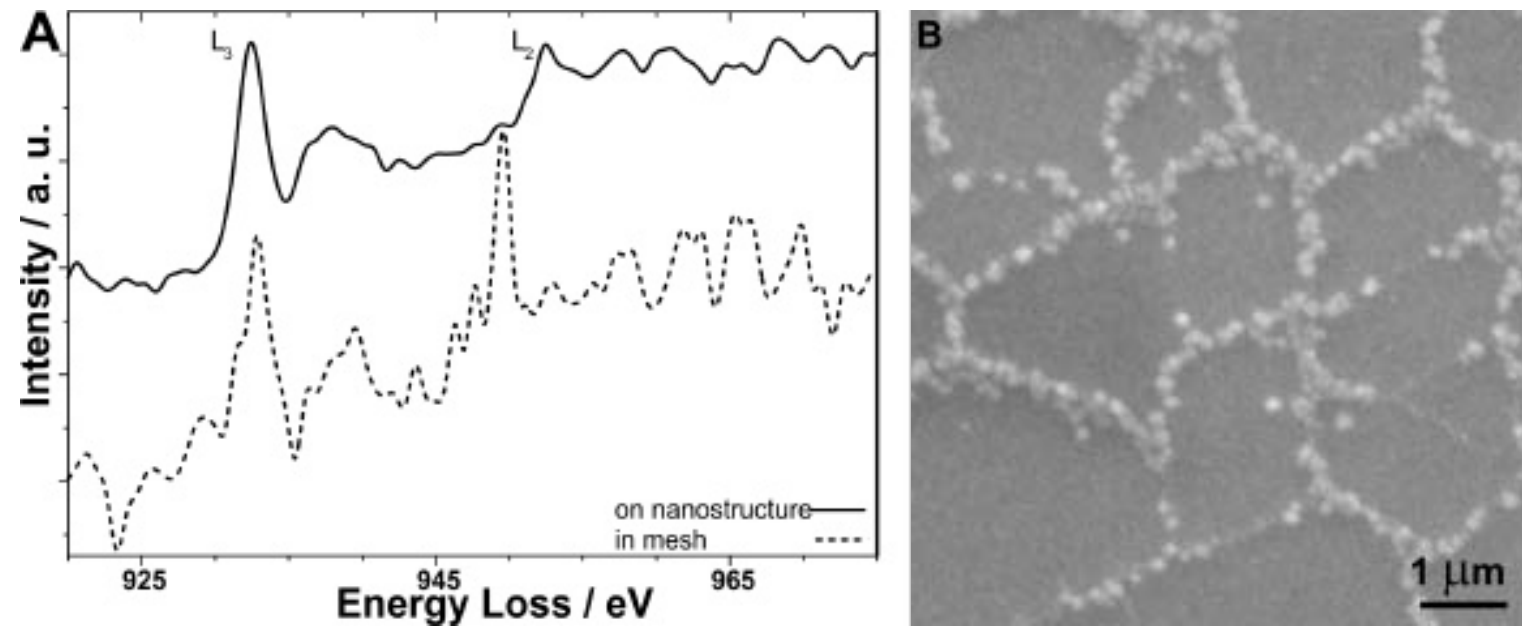

Fig.3 A: $\mathrm{Cu} \mathrm{L}_{2,3}$ ELNES from region with a nanostructure (full line) and from adjacent mesh region (broken line). B: SEM micrograph of nanostructures with aligned metal aggregates. 